\title{
Epigenetic Alterations and an Increased Frequency of Micronuclei in Women with Fibromyalgia
}

\author{
Victoria Menzies, ${ }^{1}$ Debra E. Lyon, ${ }^{1,2}$ Kellie J. Archer, ${ }^{3}$ Qing Zhou, ${ }^{3}$ Jenni Brumelle, ${ }^{4}$ \\ Kimberly H. Jones, ${ }^{4,5}$ G. Gao, ${ }^{3}$ Timothy P. York, ${ }^{6}$ and Colleen Jackson-Cook ${ }^{2,4,6}$ \\ ${ }^{1}$ Virginia Commonwealth University School of Nursing, 1100 East Leigh Street, Richmond, VA 23298-0567, USA \\ ${ }^{2}$ Massey Cancer Center, Virginia Commonwealth University, VA 23298-0037, USA \\ ${ }^{3}$ Department of Biostatistics, Virginia Commonwealth University, 830 East Main Street, Richmond, VA 23298, USA \\ ${ }^{4}$ Department of Pathology, Virginia Commonwealth University, P.O. Box 980662, Richmond, VA 23298-0662, USA \\ ${ }^{5}$ Neodiagnostix, 9700 Great Seneca Highway, Rockville, MD 20850, USA \\ ${ }^{6}$ Department of Human and Molecular Genetics, Virginia Commonwealth University, P.O. Box 980033, \\ Richmond, VA 23298-0003, USA \\ Correspondence should be addressed to Victoria Menzies; vsmenzies@vcu.edu
}

Received 30 April 2013; Accepted 14 July 2013

Academic Editor: Susan Dorsey

Copyright (C) 2013 Victoria Menzies et al. This is an open access article distributed under the Creative Commons Attribution License, which permits unrestricted use, distribution, and reproduction in any medium, provided the original work is properly cited.

Fibromyalgia (FM), characterized by chronic widespread pain, fatigue, and cognitive/mood disturbances, leads to reduced workplace productivity and increased healthcare expenses. To determine if acquired epigenetic/genetic changes are associated with FM, we compared the frequency of spontaneously occurring micronuclei (MN) and genome-wide methylation patterns in women with FM $(n=10)$ to those seen in comparably aged healthy controls $(n=42(\mathrm{MN}) ; n=8$ (methylation)). The mean (sd) MN frequency of women with FM (51.4 (21.9)) was significantly higher than that of controls $(15.8(8.5))\left(\chi^{2}=45.552 ; \mathrm{df}=1 ; P=1.49 \times 10^{-11}\right)$. Significant differences ( $n=69$ sites) in methylation patterns were observed between cases and controls considering a 5\% false discovery rate. The majority of differentially methylated (DM) sites (91\%) were attributable to increased values in the women with FM. The DM sites included significant biological clusters involved in neuron differentiation/nervous system development, skeletal/organ system development, and chromatin compaction. Genes associated with DM sites whose function has particular relevance to FM included BDNF, NAT15, HDAC4, PRKCA, RTN1, and PRKG1. Results support the need for future research to further examine the potential role of epigenetic and acquired chromosomal alterations as a possible biological mechanism underlying FM.

\section{Introduction}

Fibromyalgia (FM), which affects at least 10 million American adults [1], is a multisymptom condition resulting in not only widespread chronic pain, but also fatigue, sleep disturbances, and morning stiffness. In addition, many patients experience depression, anxiety, and dyscognition [2,3]. FM has a significant adverse impact on many individuals' physical and mental health $[4,5]$ and also leads to reduced workplace productivity and increased health care/disability expenses, with the estimated cost of FM on the US economy being reported to be $12-14$ billion dollars $[1,6]$. While the adverse impact of this condition is indisputable, its etiology remains enigmatic. Due to the lack of clarity for the underlying cause(s) of $\mathrm{FM}$, it poses a diagnostic challenge, often requiring multiple visits by specialists to render a diagnosis [7]. The lack of understanding of the biological basis of this condition also confounds our ability to develop effective interventions and/or monitor disease progression. FM has been suggested to be a complex, multifactorial trait that is influenced by age, gender (frequency is the highest in middle-aged females), and stress/trauma. Despite showing a strong familial aggregation [8-10], attempts to identify genetic factors associated with FM (primarily through polymorphism 
association studies) have yielded inconsistent results, with some investigators showing associations between FM and specific genes (including, but not limited to, genes for catechol-O-methyltransferase [11-13], serotonin-2A receptor $[14,15]$, serotonin transporter gene regulatory region $[16,17]$, dopamine D4 receptor [18], $\beta-2$ adrenergic receptor [19], gamma-aminobutyric acid receptor subunit beta-3, trace amine-associated receptor 1 , interferon-induced guanylatebinding protein 1 , regulator of $G$ protein signaling 4 , cannabinoid receptor type 1 , and glutamate receptor 4 [20]), while others failed to identify a relationship [21-25]. Since a consistent, straightforward association with a gene(s) has not yet been forthcoming, scientists have suggested that the familial influence on FM may more likely reflect a genetic susceptibility to environmental events [21, 26, 27]. For example, Klengel and Binder [28] identified differential methylation for a glucocorticoid response element (the FKBP5 gene) that resulted from the presence of both an "at-risk" allele (polymorphism) and the occurrence of childhood trauma in subjects they studied who had posttraumatic stress disorder.

Epigenetics, which refers to the process that affects gene expression independent of actual DNA sequence (such as methylation changes, histone alterations, and micro-RNA expression), has enabled scientists to conceptualize the impact of the environment upon one's genes and one's health [29]. Genes can be turned on or off and display variations in their level of expression, in part, due to epigenetic modifications [30]. Thus, epigenetics provides a biological means for understanding the molecular processes of complex biological networks that connect the brain, behavior, and health outcomes [31]. Given the overlap in symptoms and the medical/adverse social histories present in people who have FM, when compared to other conditions that have been shown to be impacted by somatic epigenetic and genetic alterations (such as depression and stress), it is plausible that similar epigenetic mechanisms may underlie the individual variability in the outcome of genetic and emotional inputs for FM.

Knowing that histone and other epigenetic modifications play a key role in establishing and maintaining chromatin structure, it follows that changes in epigenetic profiles, as a consequence of initiating events (such as stress/environmental exposure), could also lead to an increased frequency of somatic chromosomal changes. Indeed, we have shown that stress levels can impact the frequency of acquired chromosomal abnormalities by demonstrating a significantly increased frequency of somatic cell chromosomal instability in adult women who experienced childhood sexual abuse when compared to their identical cotwins who did not experience childhood sexual abuse (quantified using a micronucleus assay) [32]. Further support that chromosomal instability could arise as a downstream effect following perturbations in methylation comes from studies of individuals who have immunodeficiency, centromeric region instability, and facial anomalies (ICF) syndrome, which is an autosomal recessive condition resulting from a mutation in the methyltransferase gene $\mathrm{B}$. People with this condition have an increased frequency of acquired chromosomal abnormalities [33].
An efficient means for quantifying the frequency of acquired (somatic) chromosomal abnormalities is the cytokinesis block micronucleus (CBMN) assay, which provides information regarding the presence of chromosomal errors in somatic cells with minimal influences attributable to in vitro selective growth pressures [34]. This technique is less labor intensive than conventional cytogenetic studies but provides results that are in close agreement to those obtained using the "gold standard" of metaphase chromosomal analyses [35]. Micronuclei, which are the primary cytological structures scored in the CBMN assay, are thought to contain chromatin (from one or more chromosomes) that was not incorporated ("lagging" or "lost") into the daughter binucleates following nuclear division [34]. Micronuclei frequencies have been shown to increase with age and have been associated with a variety of health conditions $[36,37]$. However, to date, no investigators have reported the frequency of $\mathrm{MN}$ in women with FM. Based on the symptomatology and comorbidities related to this condition, we hypothesized that women with FM will have an increased frequency of acquired epigenetic and chromosomal alterations. To test this hypothesis, we initiated a pilot study to quantify chromosomal instability levels and genome-wide methylation patterns in women having FM and to compare these genetic/epigenetic values to those present in comparably aged, healthy control women.

\section{Materials and Methods}

2.1. Study Participant Ascertainment and Specimen Collection. Data for this study were obtained from a subset of participants ( $n=10)$, who were randomly recruited by mail from a larger, parent study sample of 64 women diagnosed with fibromyalgia (VCU IRB Protocol number HM12211) (Table 1). In the parent study, the participants completed a twogroup randomized, controlled, clinical trial to examine the effect of guided imagery on self-efficacy, perceived stress, pain, fatigue, depression, and neuroendocrine/neuroimmune biomarkers in women with fibromyalgia syndrome [38]. Inclusion criteria for the women having FM were age (at least 18 years old); gender (female); receipt of a physicianconfirmed diagnosis of FM based on the 1990 American College of Rheumatology criteria [39]; an ability to understand and sign the consent form; and an ability to understand/ complete the study questionnaires. Exclusion criteria for the women in the FM pilot group included the presence of other systemic rheumatologic conditions; being immunocompromised (e.g., diagnosis of HIV/AIDs); receiving corticosteroid treatments; having a major psychiatric or neurological condition that would interfere with study participation, or being pregnant. Each of the study subjects completed a self-report form to provide information regarding age, race/ethnicity, marital status, length of time since diagnosis of fibromyalgia, height and weight for calculating body mass index (BMI), and lifestyle practices such as history of smoking and alcohol use.

The healthy, comparatively aged control group of women for the MN studies $(n=42)$ were ascertained through their participation in a study of acquired genetic changes associated with normal aging, the latter of which is a twin study [40] (VCU IRB Protocol number 179). The inclusion criteria 
TABLE 1: MN frequencies and other attributes of women with FM.

\begin{tabular}{|c|c|c|c|c|c|c|c|}
\hline $\begin{array}{l}\text { Participants } \\
\text { with FM }\end{array}$ & Age & $\begin{array}{l}\text { Mean number MN } \\
\text { per } 1000 \text { binucleates }\end{array}$ & $\begin{array}{c}\text { Years since FM } \\
\text { diagnosis }\end{array}$ & Alcohol use & Smoker & Body mass index & Race \\
\hline 1009 & 48 & 32.5 & 2 & Yes & No & 45.7 & Other \\
\hline 1025 & 52 & 13.5 & 3 & Yes & Yes & 19.4 & $\mathrm{C}^{1}$ \\
\hline 1030 & 61 & 71 & 7 & No & No & 28.3 & $\mathrm{C}$ \\
\hline 1038 & 52 & 66.5 & 19 & No & No & 35.5 & $\mathrm{C}$ \\
\hline 1042 & 45 & 47.5 & 14 & No & No & 30.1 & $\mathrm{AA}^{2}$ \\
\hline 1047 & 62 & 77.5 & 19 & Yes & No & 27.1 & $\mathrm{C}$ \\
\hline 1052 & 44 & 42 & 1 & Yes & Yes & 30.5 & AA \\
\hline 1058 & 45 & 27.5 & 4 & No & No & 27.3 & AA \\
\hline 1061 & 58 & 66.5 & 2 & Yes & Yes & 21.1 & $\mathrm{C}$ \\
\hline 1066 & 56 & 69.5 & 4 & Yes & Yes & 29.0 & AA \\
\hline $\begin{array}{l}\text { FM group mean }(\mathrm{sd}) \\
(n=10)\end{array}$ & $48.2(6.7)$ & $52.1(21.9)$ & & & & $29.52(7.21)$ & \\
\hline $\begin{array}{l}\text { Control group mean (sd) } \\
(n=42)^{*}\end{array}$ & $52.0(9.8)$ & $15.8(8.5)$ & & & & & \\
\hline
\end{tabular}

* Individual data not shown.

${ }^{1} \mathrm{C}$ : Caucasian (white); ${ }^{2}$ AA: African American (Black).

for this subset of control subjects were gender (female) and age (range from 36 to 69 years old), with all people from the previous study who met the criteria being included as controls for the current study to avoid sampling biases. The control cohort of women included both cotwin pairs $(n=30$ women or 15 cotwin pairs) and single twins, whose cotwin did not participate in this normal aging study $(n=12$ women). Due to cost limitations, DNA methylation studies were limited to a subset $(n=8)$ of the control women. This subset of women was randomly selected and included 8 unrelated females (no cotwins). All of the control women self-reported their age, race/ethnicity, and lifestyle practices, such as history of smoking and alcohol use.

2.2. Biological Assays. Following the collection of the blood specimens from the patient and control women, the cells were processed to obtain binucleates for the micronuclei studies and DNA for the methylation studies as described in the following section.

2.2.1. Micronucleus Assay. Lymphocytes were collected using Histopaque-1077 (Sigma), stimulated with the mitogen phytohemagglutinin, established in culture, and blocked at cytokinesis according to the protocol of Fenech [35]. Briefly, cytochalasin B (3.0 $\mu \mathrm{g} / \mathrm{mL}$; Sigma, 14930-92-2) was added to the cells 44 hours after culture initiation. At 72 hours, binucleate interphase cells were harvested using standard techniques, which included a 10-minute incubation in hypotonic solution $(0.075 \mathrm{M} \mathrm{KCl})$ and serial fixation (three times using a $3: 1$ methanol: acetic acid solution). Slides were made following standard procedures. Micronuclei were visualized following Giemsa staining ( $4 \%$ Harleco Giemsa solution) and identified according to the criteria established by Fenech [35] (Figure 1). The frequencies of micronuclei observed in the cytochalasinB-blocked binucleated cells of the women with FM and the healthy control women were calculated by averaging the values obtained from two replicate scores (1000 binucleates were evaluated from two independent areas of the slide) for a total of 2000 binucleates that were evaluated per study participant.

2.2.2. DNA Isolation and Genome-Wide Methylation Assay. Genomic DNA was isolated from whole blood according to standard methods using the Puregene DNA isolation kit (Qiagen). An aliquot (500 ng per study participant) of DNA was then sent to Hudson Alpha Institute for Biotechnology for bisulfite conversion, using standard methods (Zymo Research EZ Methylation Kit) and genome-wide methylation pattern assessment, using the $450 \mathrm{~K}$ HumanMethylation Chip, according to the manufacturer's protocol (Illumina). The $450 \mathrm{~K}$ HumanMethylation Chip interrogates 485,764 genome-wide targets. Intensity data from the scanned arrays were imported into Illumina's GenomeStudio software and the minfi Bioconductor package in the R programming environment to obtain the $\beta$ values for each probe.

\subsection{Statistical Analyses}

2.3.1. Micronuclei Analyses. To test the hypothesis that women with FM have an increased frequency of acquired chromosomal alterations, the frequency of MN was compared between the women diagnosed with FM and the control group women. Given that a portion of the healthy controls were cotwins, a mixed effect model was used to control for familial relationships. MN frequency comparisons between cases and controls were adjusted for age since several studies have demonstrated increases in $\mathrm{MN}$ frequency with age [37, 40-42]. The independent effect of age on MN was evaluated using a Spearman correlation. Additional environmental effects were considered that have previously been 


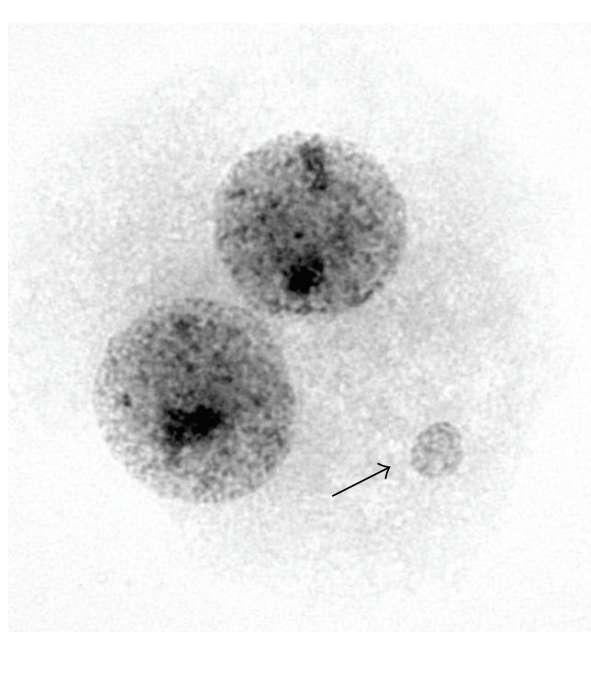

(a)

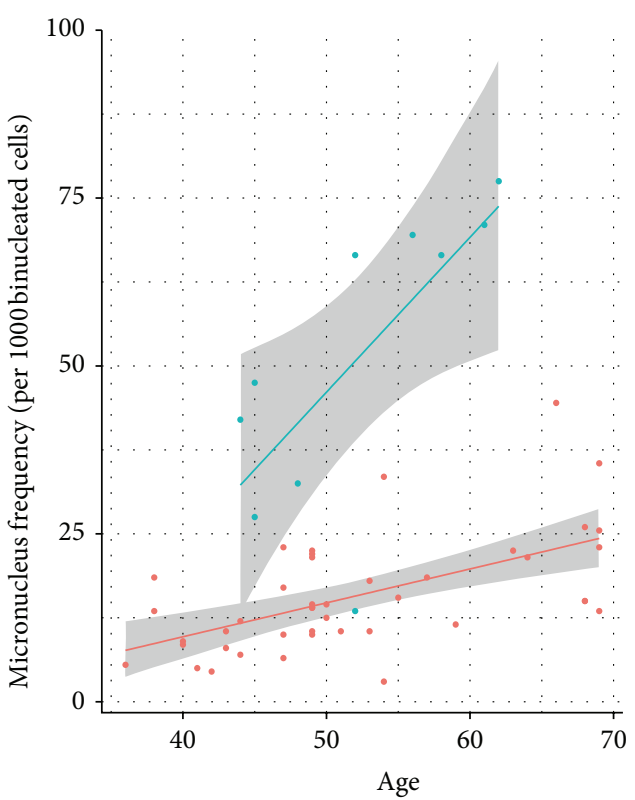

Fibromyalgia

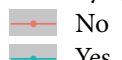

(b)

FIGURE 1: Increased frequency of micronuclei (MN) in women with FM. (a) A representative cell blocked at the cytokinesis phase of mitosis is shown. Karyokinesis has been completed, resulting in 2 daughter binucleates having a single MN (arrow). (b) The frequency of MN ( $y$-axis) showed the expected age-related increase (age on $x$-axis) for both controls (Spearman's rho $=0.579)$ and patients having FM (Spearman's rho $=0.717$ ). The least squares regression line with corresponding $95 \%$ confidence interval (grey region) is shown for each group. After controlling for age effects, the frequencies of MN were found to be significantly higher in the women with FM compared to healthy controls $\left(P=1.49 \times 10^{-11}\right)$.

shown to influence micronuclei frequencies. These included body mass index, alcohol use, and smoking in the women having FM [40]. However, given that values for body mass index, alcohol use, and smoking were not available for the controls (in a format comparable to those for cases), these variables were only assessed for the women with FM.

2.3.2. Genome-Wide Methylation Analyses. Because the $450 \mathrm{~K}$ HumanMethylation assay includes both the Infinium I design (which includes two probes for interrogating a CpG site) and the Infinium II design (which includes only one probe), the GC content was plotted separately by Infinium design type for the fully methylated sample, for which all CpG sites are expected to have consistently high $\beta$ values [43]. Based on these results, probes having a GC content greater than 40 were excluded from further analysis to ensure that the results would not be biased by the "GC" content of the underlying sequence. In addition, since the performance of probes containing single nucleotide polymorphisms (SNPs) can be variable, probes containing SNPs that were present within 10 bases of the target site were also excluded [43]. Because the expression value, $\beta$, reported for each $\mathrm{CpG}$ site represents "proportion methylated" which is constrained to an interval value from 0 to 1 , where a $\beta$ of 1 indicates complete methylation and 0 indicates no methylation, the expression values were transformed using the logit transformation
$[M=\log (\beta /(1-\beta)]$ to promote normality [44]. Prior to the logit transformation, imputation was completed (0.001 for $\beta$ values that were 0 and 0.999 for $\beta$ values that were 1) to avoid nonexistent $M$ values. To adjust for the observation that $\beta$ values from the Infinium II designed probes have a compressed range compared to the $\beta$ values from the Infinium I design $[43,45,46]$, the peak-correction method was applied to the logit transformed $\beta$ values for the Infinium II designed probes [46].

Statistical analyses were then performed on the peak corrected logit transformed $\beta$ values from the patient and control samples. For each CpG site, differential methylation between specimens collected from women with FM and controls was tested using the moderated $t$-test in the limma Bioconductor package $[51,52]$ in the $\mathrm{R}$ programming environment [53]. To adjust for the multiple hypothesis tests, the $P$ values were used to estimate the false discovery rate (FDR) following Benjamini and Hochberg's [54] method. The DAVID gene functional classification tool [55] was used to identify biological relationships among the differentially methylated sites.

\section{Results}

3.1. Micronucleus Assay. As expected, the frequency of MN was correlated with age in both the women having FM 
TABLE 2: Genes associated with sites differentially methylated in patients with fibromyalgia.

\begin{tabular}{l} 
Gene name (abbreviation) \\
\hline AXL receptor tyrosine kinase (AX \\
N-Acetyltransferase 15 (NAT15) \\
Solute carrier family 17, member 9 \\
(SLC17A9)
\end{tabular}

Brain-derived neurotrophic factor (BDNF)

Mahogunin ring finger 1, E3 ubiquitin protein ligase (MGRN1)

Plasma glutamate carboxypeptidase (PGCP)

Protein kinase C, alpha (PRKCA)

Gamma-glutamyltransferase 1 (GGT1)

Reticulon 1 (RTN1)

NFXL1 nuclear transcription factor, X-box binding-like 1 (NFXL1)

Heat shock $70 \mathrm{kDa}$ protein 8 (HSPA8)

Polymeric immunoglobulin receptor (PIGR)

Benzodiazepine receptor (peripheral) associated protein 1 (BZRAP1)

Transmembrane protein 91 (TMEM91)

Neuron-derived orphan receptor 1 (NR4A3)

$\mathrm{V}$-set and immunoglobulin domain containing 10 (VSIG10)

Potassium voltage-gated channel subfamily $\mathrm{H}$ member 7 (KCNH7)

$\mathrm{V}$-set and transmembrane domain containing 2A (VSTM2A)

Ephrin type-A receptor 2 (EPHA2)

Patched 2 (PTCH2)

Histone deacetylase 4, (HDAC4)

ADP-ribosylarginine hydrolase (ADPRH)

Fasciculation and elongation protein zeta 1 (zygin I) (FEZ1)
Description of function $^{1}$
Involved in stimulation of cell proliferation and aggregation; induced
by TGF- $\beta 1$ /inflammation; and can block cytokine production

by TGF- $\beta 1$ /inflammation; and can block cytokine production
Histone acetyltransferase; mediates acetylation of free histone H4; also required for normal chromosomal segregation during anaphase

Vesicular nucleotide transporter involved in exocytosis of ATP in secretory vesicles (synaptic vesicles)

Involved in the regulation of stress response and in the biology of mood disorders; major regulator of synaptic transmission and plasticity at adult synapses in many regions of the CNS; contributes to several adaptive neuronal responses including long-term potentiation, long-term depression, certain forms of short-term synaptic plasticity, and homeostatic regulation of intrinsic neuronal excitability

Mediates monoubiquitination at multiple sites; plays a role in the regulation of endosome-to-lysosome trafficking.

Involved in hydrolysis of circulating peptides Location $^{2}$

19: 41731934

16: 3507460

20: 61584072

Involved in diverse cell signaling pathways; activated by calcium; associated with cancer; posttraumatic stress syndrome; emotional memory formation; and aging

Function not clear and may vary; has been associated with arterial stiffness and coronary artery disease [47]

Involved in secretion or membrane trafficking in neuroendocrine cells; associated with neurological diseases and cancer

Integral to the nucleus and membrane

11: 27740495

Member of the heat shock protein family, functions as a chaperone and facilitates protein folding

Member of the immunoglobulin superfamily that facilitates expression of immunoglobulins A and M; regulated by cytokines

An adaptor molecule known to regulate synaptic transmission [48]

In vivo function not clearly established

Target of $\beta$-andrenergic signaling in skeletal muscle [49]

In vivo function not clearly established

16: 4714733

8: 97657294

17: 64787379

22: 24989248

14: 60097209

4: 47917042

11: 122933028

1: 207103660

17: 56401800

19: 41882253

9: 102588232

12: 118541722

Involved in regulating neurotransmitter release, heart rate, insulin secretion, neuronal excitability, epithelial electrolyte transport, smooth muscle contraction, and cell volume.

In vivo function not clearly established

2: 163695111

7: 54609587

Protein-tyrosine kinase family member; implicated in mediating developmental events, particularly in the nervous system.

A transmembrane receptor of the patched gene family; may function as a tumor suppressor in the hedgehog signaling pathway; has been associated with several different types of cancer

Responsible for the deacetylation of the core histones; gives tag for epigenetic repression; plays important role in transcriptional regulation, cell cycle progression, and developmental events; also involved in muscle maturation through interaction with the myocyte enhancer factors

Catalyzes removal of mono-ADP-ribose from arginine residues of proteins in the ADP-ribosylation cycle.

Involved in normal axonal bundling and elongation within axon bundles; may also function in axonal outgrowth.
1: 16482553

1: 45297445

2: 240044021

3: 119299162

11: 125365478 
TABLE 2: Continued.

Gene name (abbreviation)
Superoxide dismutase 3, extracellular
(SOD3)
Transcription factor AP-2 alpha 2 (TFAP2A)
Odz, odd Oz/ten-m homolog 3 (ODZ3)
Ephrin type A receptor 5 (EPHA5)
Suppressor of fused homolog (Drosophila)
(SUFU)
Rh family, C glycoprotein (RHCG)
DNAJ (Hsp40) homolog, subfamily C,
member 17 (DNAJC17)
Autism susceptibility candidate 2 (AUTS2)
Deleted in lymphocytic leukemia, 7
(DLEU7)

SH2B adaptor protein 2 (SH2B2)

Alpha-kinase 3 (ALPK3)

VENT homeobox (VENTX)

Lymphocyte antigen 6 complex, locus G5C (LY6G5C)

Primary ciliary dyskinesia protein 1 (PCDP1)

Protein kinase, cGMP-dependent, type I (PRKG1)

MAP7 domain containing 2 (MAP7D2)

Carboxypeptidase M (CPM)

Growth differentiation factor 1

(GDF1)/LAG1 longevity assurance homolog 1 (LASS1)

Ubiquitin-like, containing PHD and RING finger domains, 1 (UHRF1)

Anoctamin 3 (AN03)

Homeobox protein Hox-A7 (HOXA7)

Transmembrane protein 44 (TMEM44)

Description of function $^{1}$
Antioxidant enzyme thought to protect the brain, lungs, and other
tissues from oxidative stress.

Transcription factor; activates the transcription of some genes while inhibiting the transcription of others

May function as a cellular signal transducer Location $^{2}$

4: 24801801

6: 10420079

4: 183370512

Mediates developmental events, particularly in the nervous system; plays a role in synaptic plasticity in adult brain through regulation of synaptogenesis; also mediates communication between pancreatic

4: 66535145 islet cells to regulate glucose-stimulated insulin secretion

Plays a role in pattern formation and cellular proliferation during development; encodes a negative regulator of the hedgehog signaling pathway

Functions as an electroneutral and bidirectional ammonium transporter

Heat shock protein homolog

15: 41062113

Function not fully known; deletions of this gene have been associated with autism and intellectual disability

In vivo function not clearly established

7: 70158761

13: 51417846

Involved in multiple signaling pathways; may function as a negative regulator of cytokine signaling; suppresses PDGF-induced

mitogenesis: may induce cytoskeletal reorganization via interaction with VAV3

Recognizes phosphorylation sites (alpha-helical conformation); may play a role in cardiomyocyte differentiation

May function as a transcriptional repressor and influence mesodermal patterning and hemopoietic stem cell maintenance Located in the major histocompatibility complex (MHC) region on chromosome 6; may be involved in signal transduction or hematopoietic cell differentiation

May function in ciliary motility

10: 104393081

15: 90039613

7: 101934892

15: 85360691

10: 135050326

6: 31649619

2: 120301847

Involved in signal transduction processes in diverse cell types; plays role in regulating cardiovascular and neuronal functions and in relaxing smooth muscle tone, preventing platelet aggregation, and modulating cell growth

$\mathrm{X}$-linked imprinted gene that may affect sex-specific brain function and/or sex-dependent neurobiological traits [50]

Associated with monocyte to macrophage differentiation; may play role in control of peptide hormone and growth factor activity at the cell surface and in the membrane-localized degradation of extracellular proteins

Member of TGF-beta superfamily; may function in regulation of cell growth and differentiation in embryonic and adult tissues and neural development in later embryogenesis; may be involved in aging

Recruits a histone deacetylase to regulate gene expression; involved in G1/S transition and functions in the p53-dependent DNA damage checkpoint

May act as a calcium-activated chloride channel

DNA-binding transcription factor that may regulate gene expression, morphogenesis, and differentiation

11: 26353723

7: 27196790

Enriched in the bottom portion of taste buds 
TABLE 2: Continued.

\begin{tabular}{ll}
\hline Gene name (abbreviation) & Description of function $^{1}$ \\
\hline & $\begin{array}{l}\text { Voltage-gated potassium channel required for repolarization phase of } \\
\text { the cardiac action potential; mutations associated with hereditary }\end{array}$ \\
$\begin{array}{l}\text { Potassium voltage-gated channel, KQT-like } \\
\text { subfamily, member 1 (KCNQ1) }\end{array}$ & $\begin{array}{l}\text { long QT syndrome 1, the Jervell and Lange-Nielsen syndrome, and } \\
\text { familial atrial fibrillation; exhibits tissue-specific imprinting; located } \\
\text { in a region associated with the Beckwith-Wiedemann syndrome }\end{array}$ \\
$\begin{array}{ll}\text { Polymerase I and transcript release factor 1 } \\
\text { (PTRF) }\end{array}$ & $\begin{array}{l}\text { Regulates rRNA transcription; thought to modify } \\
\text { lipid metabolism and insulin-regulated gene expression }\end{array}$ \\
\hline
\end{tabular}

${ }^{1}$ Functional descriptions obtained from Gene Cards and/or Wikipedia summations and indicated references.

${ }^{2}$ Chromosome: starting nucleotide position.

TABLE 3: Cluster analysis of genes having significantly different methylation patterns in women with FM.

\begin{tabular}{lcl}
\hline Genetic ontology/keyword term & No. of Genes & $P$ value \\
\hline Anatomical structure development & 17 & 0.0002 \\
System development & 16 & 0.0003 \\
Developmental process & 18 & 0.0008 \\
Multicellular organismal development & 17 & 0.0009 \\
Polymorphism & 41 & 0.0011 \\
Sequence variant & 42 & 0.0012 \\
Domain: fibronectin type III 1,2 & 4 & 0.0045 \\
Glycosylation site & 20 \\
Neuron differentiation & $\mathbf{6}$ \\
Nervous system development & $\mathbf{9}$ \\
Protein metabolic process & 15 \\
Autophosphorylation & 3 \\
Glycoprotein & 20 & 0.0047 \\
Skeletal system development & 5 & $\mathbf{0 . 0 0 4 8}$ \\
Organ development & 11 & 0.0053 \\
\hline
\end{tabular}

$(r=0.717 ; P=0.021)$ and the healthy controls $(r=0.579$; $P=4.79 \times 10^{-5}$ ) (Figure 1). After controlling for the effect of age and cotwin status, a significantly increased frequency of MN was observed in the women having FM (51.4 (21.9) (mean (sd)) per 1000 binucleates) compared to controls (15.8 (8.5) (mean (sd)) per 1000 binucleates) $\left(\chi^{2}=45.6\right.$; $\mathrm{df}=1$; $P=1.49 \times 10^{-11}$ ) (Figure 1). The increased levels of $\mathrm{MN}$ in the women having FM were not significantly correlated with their body mass index (range from 19.44 to 45.70; mean (sd) was 29.52 (7.21); $P=0.997$ ), smoking history (4 smokers; 6 nonsmokers; $P=0.75$ ), or alcohol use ( 6 consumers; 4 nonconsumers; $P=0.93$ ). To assess if there might be a cumulative biological effect associated with experiencing symptoms associated with FM, we compared MN frequencies for the case subjects $(n=10)$ with the total number of years that had lapsed since these women received their diagnosis of FM. While there was a trend toward a positive correlation between a woman's MN frequency and the number of years since she was diagnosed with FM (ranged from 2 to 19 years), this relationship did not reach significance in this small pilot study $(P=0.134)$ (Table 1$)$.

3.2. Genome-Wide Methylation Assay. After completion of the quality control assessments that were performed to remove any potential biases associated with probe sequence length, probe GC content, and inclusion of SNPs [56], a total of 381,989 CpG sites were retained. From these, a total of 69 sites were determined to be differentially methylated (DM) between the patients who have FM and the healthy controls, with 63 of these DM sites having higher values in the patients with FM and 6 having lower values (Figure 2). These $69 \mathrm{DM}$ sites included CpG islands (46.4\%); north shores $(20.3 \%)$; south shores $(8.7 \%)$; as well as north $(4.3 \%)$ and south $(1.4 \%)$ shelves and sites that were not annotated into the previously noted categories $(18.8 \%)$. The DM sites were localized to 47 different genes (Table 2), with 3 genes having multiple sites identified (N-acetyltransferase 15 gene (NAT15) had 4 DM sites; DNAJ (Hsp40) homolog, subfamily C, member 17 (DNAJC17) had 2 DM sites; and SLC17A9 and 2 DM sites). An assessment of potential biologically related clusters of DM sites resulted in the recognition of 15 groups, including gene clusters involved in neuron differentiation and nervous system development (Table 3).

\section{Discussion}

While the sample size in this pilot study is small, the $\mathrm{MN}$ frequency patterns of both the case and control women showed an age-related increase, which is a finding that is in 


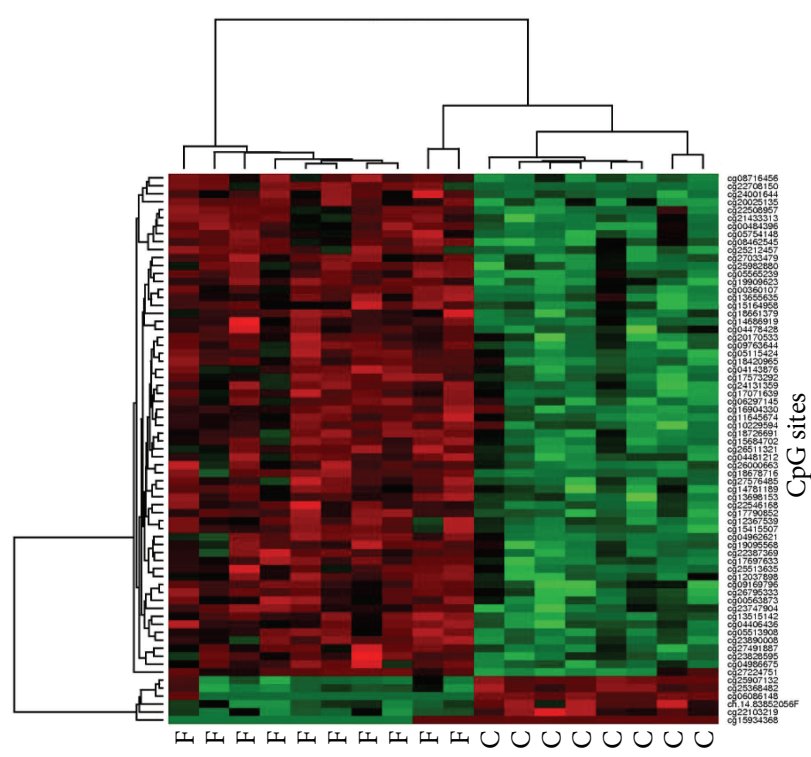

Study subjects

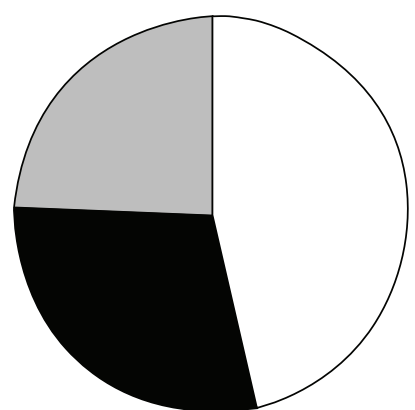

FM: controls

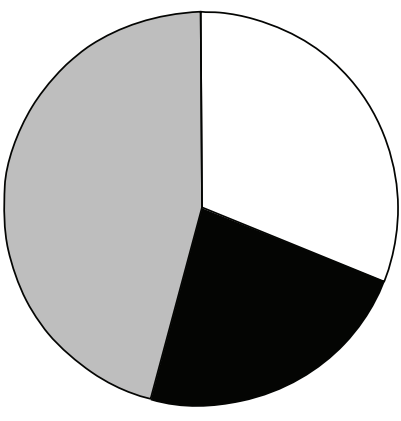

$450 \mathrm{~K}$ array

$\square$ Islands

- Shores

$\square$ Distant

(a)

(b)

FIGURE 2: Sites having significantly different methylation patterns in women with FM compared to healthy controls. (a) A heatmap shows the $69 \mathrm{DM}$ sites detected in the study. The women who have FM (F) are grouped on the left of the figure, with controls (C) being grouped on the right. Each row (CpG site) is scaled to represent methylation where lower levels are represented in green while higher levels are represented in red. The majority of significant differences resulted from women with FM (upper left red cluster) having higher levels compared to controls (upper cluster), with a smaller cluster (lower left green) showing lower methylation levels in the cases with FM compared to healthy controls. (b) Pie charts showing the percentage of DM sites according to their relationship to CpG islands. Nearly half (46.4\%) of the sites that were significantly different between the women with FM compared to controls were localized to CpG islands (top pie chart), whereas CpG islands account for only about one third (31\%) of the sites interrogated on the $450 \mathrm{~K}$ HumanMethylation array (bottom pie chart). The proportion of DM sites localized to shores that were recognized in this study (29\%) closely paralleled the proportion of sites localized to shores that are interrogated in the array (23\%). Sites associated with a location distant to CpG islands accounted for $24.5 \%$ of the DM sites identified in this study but accounted for $46 \%$ of the total sites interrogated on the array.

agreement with the age-related increase that has consistently been reported in larger studies [40, 41]. Interestingly, the mean frequency of MN in the women with FM was 3.26fold higher than the level seen in the healthy controls. In comparison, patients who have cancer have been noted to have 1.37- to 3.13-fold higher frequencies of MN when compared to healthy controls $[57,58]$. Given that the risk for cancer has been shown to be predicted by MN levels [37, 41, 58], the results of this preliminary data, if confirmed, suggest that $\mathrm{MN}$ frequency assessments may be useful for evaluating/diagnosing women with FM. Indeed, recent assessments of $\mathrm{MN}$ frequencies in people evaluated from various areas of biobehavioral science have shown increased levels of acquired chromosomal instability (assessed using MN frequencies) in adult women who experienced childhood sexual abuse [32]; patients who have neurodegenerative conditions, such as Alzheimer's disease and Parkinson's disease [59]; and adults with type 2 diabetes and cardiovascular disease [60]. The presence of acquired chromosomal instability, which could lead to somatic tissue mosaicism, has been conjectured to occur as a global biological process that affects many tissues and contributes to the development of several conditions, including (but not limited to) autism, schizophrenia, autoimmune diseases, and Alzheimer's disease [61]. Given that several of these conditions are age related, one could speculate that there may be a "threshold" level of chromosomal instability required for eliciting a biological consequence. Factors contributing to $\mathrm{MN}$ formation are multifold and include both genetic [40] and environmental influences [37, 40, 41]. Environmental exposures that have been shown to increase the frequency of MN include, but are not limited to, diet (especially folate deficiency) [41, 62, 63], hormone levels [64], and exposures to substances/occupational hazards [37]. The biological means whereby these genetic/environmental influences lead to acquired chromosomal instability are likely to be varied but have been noted to reflect the chromatin 
conformation of the chromosomes [65]. One can speculate that alterations in chromatin conformation, which are likely to arise (at least in part) from epigenetic changes, may compromise the ability of the chromosomes to align, attach to mitotic spindle fibers, and/or separate, thereby leading to their increased frequency of abnormalities [66]. In turn, the presence of acquired chromosomal abnormalities could lead to additional epigenetic alterations.

While limited in number, studies performed to assess the effect of methylation on chromosome segregation have consistently shown an increase in the frequency of cytogenic abnormalities associated with perturbations in the methylation status of chromosomes [67]. In this study, it is interesting to note that DM sites were identified for genes having a function related to chromatin compaction and/or segregation (NAT15; HDAC4; UHRF1). For example, DM sites were observed for the NAT15 gene, which is a gene that has been identified to play an important role in normal chromosomal segregation during anaphase [68]. While the results of the genome-wide methylation patterns evaluated in this study are preliminary, it is exciting to note that several of the sites that were DM between the women with FM and controls were localized to genes that have functional relevance to the symptoms seen in patients with FM. Of particular interest was the observation of a significant difference in the methylation pattern of the BDNF gene between patients with FM and controls. The BDNF gene has been noted to play an important neuromodulatory role in pain transduction (eliciting hyperalgesia) [69-71] and has also been recognized as a contributor to learning and memory $[72,73]$. A second gene of apparent relevance with which a DM site was associated was the protein kinase $\mathrm{C}$, alpha gene (PRKCA) (Table 3). This gene, which is involved in cell signaling pathways, has been associated with emotional memory formation, posttraumatic stress syndrome, cancer, and aging. A third gene of particular interest that had a DM site is Reticulon 1 (RTN1). RTN1 has been associated with neurological diseases (and cancer) and is thought to influence membrane trafficking in neuroendocrine cells. Overall, genes with which DM sites were associated include (but are not limited to) those having functions in chromatin compaction (NAT15; HDAC4; UHRF1); DNA damage/repair or chromosomal segregation (SOD3; UHRF1; NAT15); muscle contraction (NR4A3; HDAC4; FEZ1; PRKG1; KCNH7); axonal bundling/outgrowth (FEZ1); cell signaling in muscle (NR4A3; PRKG1); neuronal excitability/synaptic transmission (BDNF; BZRAP1; EPHA5; KCNH7); muscle maturation (HDAC4); response to oxidative stress (SOD3); and inflammatory processes (AXL; SH2B2). However, since two of the significant biological clusters that were identified (Table 3) were for polymorphisms and sequence variants, it is important to recognize that this is a pilot study and that a larger number of individuals will need to be evaluated to allow one to determine the extent, if consistently present, of $\mathrm{DM}$ on the development or severity of symptoms associated with FM.

The results of genome-wide methylation studies have provided insight regarding the role of genes and environmental influences for a variety of conditions, with many of these investigations focusing on the areas of cancer and psychiatric conditions [74, 75]. However, the epigenomes of diseases causing chronic pain have been less extensively evaluated. In their study of rheumatoid arthritis, Nakano et al. [76] observed several DM sites between patients who have rheumatoid arthritis and controls. They also identified distinct epigenomic signatures when comparing patterns from patients with rheumatoid arthritis and osteoarthritis. Akin to the results of this pilot study, the findings of their investigation led to the recognition of perturbations in the methylation status of several genes having functions related to the symptoms associated with rheumatoid arthritis. Thus, the use of genome-wide epigenetic assessment seems to be a promising tool for evaluating a broad spectrum of conditions, including those associated with chronic pain.

\section{Conclusion}

In summary, the results of this pilot study suggest that chromosomal instability and alterations in methylation are present in women with FM. If these results can be confirmed, they could provide a basis for improving our understanding of the biological changes leading to the development of FM and may provide a basis for stratifying patients based on their epigenomic and symptom patterns. Moreover, since epigenetic changes demonstrate plasticity [77], the recognition of consistent epigenetic alterations associated with FM could provide a means for developing future therapeutic approaches to reverse these changes, with a goal of alleviating symptoms in people who have FM.

\section{Acknowledgments}

This work was supported in part by grants from the National Institute of Nursing Research through Grant (P30NR011403, Grap (PI)) and the National Institute of Environmental Health (R01 ES12074 C Jackson-Cook (PI)). Its contents are solely the responsibility of the authors and do not necessarily represent the official views of the NIEHS, NIH.

\section{References}

[1] About Fibromyalgia: Economic Burden, 2013, http://fmaware.org/ site/PageServerc145.html?pagename $=$ fibromyalgia_fmFactSheet.

[2] M. A. Fitzcharles and M. B. Yunus, "The clinical concept of fibromyalgia as a changing paradigm in the past 20 years," Pain Research and Treatment, vol. 2012, Article ID 184835, 8 pages, 2012.

[3] D. A. Williams, D. J. Clauw, and J. M. Glass, "Perceived cognitive dysfunction in fibromyalgia syndrome," Journal of Musculoskeletal Pain, vol. 19, no. 2, pp. 66-75, 2011.

[4] L. Dreyer, S. Kendall, B. Danneskiold-Samsøe, E. M. Bartels, and H. Bliddal, "Mortality in a cohort of Danish patients with fibromyalgia: increased frequency of suicide," Arthritis and Rheumatism, vol. 62, no. 10, pp. 3101-3108, 2010.

[5] F. Wolfe, A. L. Hassett, B. Walitt, and K. Michaud, "Mortality in fibromyalgia: a study of 8,186 patients over thirty-five years," Arthritis Care and Research, vol. 63, no. 1, pp. 94-101, 2011.

[6] D. J. Wallace, "Is there a role for cytokine based therapies in fibromyalgia," Current Pharmaceutical Design, vol. 12, no. 1, pp. 17-22, 2006. 
[7] T. M. Brown, S. Garg, A. B. Chandran, M. McNett, S. L. Silverman, and N. Hadker, "The impact of 'best-practice' patient care in fibromyalgia on practice economics," The Journal of Evaluation in Clinical Practice, vol. 18, no. 4, pp. 793-798, 2012.

[8] L. M. Arnold, J. Fan, I. J. Russell et al., "The fibromyalgia family study: a genome-wide linkage scan study," Arthritis \& Rheumatism, vol. 65, no. 4, pp. 1122-1128, 2013.

[9] K. C. Light, A. T. White, S. Tadler, E. Iacob, and A. R. Light, "Genetics and gene expression involving stress and distress pathways in fibromyalgia with and without comorbid chronic fatigue syndrome," Pain Research and Treatment, vol. 2012, Article ID 427869, 13 pages, 2012.

[10] L. A. Bradley, "Pathophysiology of fibromyalgia," American Journal of Medicine, vol. 122, no. 12, supplement, pp. S22-S30, 2009.

[11] S. Gürsoy, E. Erdal, H. Herken, E. Madenci, B. Alaşehirli, and N. Erdal, "Significance of catechol-O-methyltransferase gene polymorphism in fibromyalgia syndrome," Rheumatology International, vol. 23, no. 3, pp. 104-107, 2003.

[12] J.-K. Zubieta, M. M. Heitzeg, Y. R. Smith et al., "COMT val158 genotype affects $\mu$-opioid neurotransmitter responses to a pain stressor," Science, vol. 299, no. 5610, pp. 1240-1243, 2003.

[13] H. Cohen, L. Neumann, Y. Glazer, R. P. Ebstein, and D. Buskila, "The relationship between a common catechol-O-methyltransferase (COMT) polymorphism val158met and fibromyalgia," Clinical and Experimental Rheumatology, vol. 27, no. 5, supplement 56, pp. S51-S56, 2009.

[14] B. Bondy, M. Spaeth, M. Offenbaecher et al., "The T102C polymorphism of the 5-HT2A-receptor gene in fibromyalgia," Neurobiology of Disease, vol. 6, no. 5, pp. 433-439, 1999.

[15] S. Gürsoy, E. Erdal, H. Herken, E. Madenci, and B. Alaşehirli, "Association of T102C polymorphism of the 5-HT2A receptor gene with pyschiatric status in fibromyalgia syndrome," Rheumatology International, vol. 21, no. 2, pp. 58-61, 2001.

[16] M. Offenbaecher, K. Glatzeder, and M. Ackenheil, "Self-reported depression, familial history of depression and fibromyalgia (FM), and psychological distress in patients with FM," Zeitschrift fur Rheumatologie, vol. 57, supplement 2, pp. 94-96, 1998.

[17] S. Gursoy, "Absence of association of the serotonin transporter gene polymorphism with the mentally healthy subset of fibromyalgia patients," Clinical Rheumatology, vol. 21, no. 3, pp. 194-197, 2002.

[18] D. Buskila, H. Cohen, L. Neuman, and R. P. Ebstein, "An association between fibromyalgia and the dopamine $\mathrm{D} 4$ receptor exon III repeat polymorphism and relationship to novelty seeking personality traits," Molecular Psychiatry, vol. 9, no. 8, pp. 730727, 2004.

[19] G. Vargas-Alarcón, J.-M. Fragoso, D. Cruz-Robles et al., "Association of adrenergic receptor gene polymorphisms with different fibromyalgia syndrome domains," Arthritis and Rheumatism, vol. 60, no. 7, pp. 2169-2173, 2009.

[20] S. B. Smith, D. W. Maixner, R. B. Fillingim et al., "Large candidate gene association study reveals genetic risk factors and therapeutic targets for fibromyalgia," Arthritis and Rheumatism, vol. 64, no. 2, pp. 584-593, 2012.

[21] A. R. Light, L. Bateman, D. Jo et al., "Gene expression alterations at baseline and following moderate exercise in patients with Chronic Fatigue Syndrome and Fibromyalgia Syndrome," Journal of Internal Medicine, vol. 271, no. 1, pp. 64-81, 2012.

[22] B. Frank, B. Niesler, B. Bondy et al., "Mutational analysis of serotonin receptor genes: HTR3A and HTR3B in fibromyalgia patients," Clinical Rheumatology, vol. 23, no. 4, pp. 338-344, 2004.

[23] B. Tander, S. Gunes, O. Boke et al., "Polymorphisms of the serotonin-2A receptor and catechol-O-methyltransferase genes: a study on fibromyalgia susceptibility," Rheumatology International, vol. 28, no. 7, pp. 685-691, 2008.

[24] M. von der Hagen, J. Schallner, A. M. Kaindl et al., "Facing the genetic heterogeneity in neuromuscular disorders: linkage analysis as an economic diagnostic approach towards the molecular diagnosis," Neuromuscular Disorders, vol. 16, no. 1, pp. 4-13, 2006.

[25] B. I. Nicholl, K. L. Holliday, G. J. Macfarlane et al., "No evidence for a role of the catechol-O-methyltransferase pain sensitivity haplotypes in chronic widespread pain," Annals of the Rheumatic Diseases, vol. 69, no. 11, pp. 2009-2012, 2010.

[26] S. Katoa, Y. Katoa, T. Nakamuraa et al., "Genetic deficiency of carnitine/organic cation transporter 2 (slc22a5) is associated with altered tissue distribution of its substrate pyrilamine in mice," Biopharmaceutics and Drug Disposition, vol. 30, no. 9, pp. 495-507, 2009.

[27] J. N. Ablin, H. Cohen, and D. Buskila, "Mechanisms of disease: genetics of fibromyalgia," Nature Clinical Practice Rheumatology, vol. 2, no. 12, pp. 671-678, 2006.

[28] T. Klengel and E. B. Binder, "Allele-specific epigenetic modification: a molecular mechanism for gene-environment interactions in stress-related psychiatric disorders?" Epigenomics, vol. 5, no. 2, pp. 102-109, 2013.

[29] M. Szyf, "How do environments talk to genes?" Nature Neuroscience, vol. 16, no. 1, pp. 2-4, 2013.

[30] D. L. Molfese, "Advancing neuroscience through epigenetics: molecular mechanisms of learning and memory," Developmental Neuropsychology, vol. 36, no. 7, pp. 810-827, 2011.

[31] G. M. Slavich and S. W. Cole, "The emerging field of human social genomics," Clinical Psychological Science, vol. 1, no. 3, pp. 331-348, 2013.

[32] T. P. York, J. Brumelle, J. Juusola et al., "Increased frequency of micronuclei in adults with a history of childhood sexual abuse: a discordant monozygotic twin study," PLoS One, vol. 8, no. 1, Article ID e55337, 2013.

[33] T. Kondo, M. P. Bobek, R. Kuick et al., "Whole-genome methylation scan in ICF syndrome: hypomethylation of non-satellite DNA repeats D4Z4 and NBL2," Human Molecular Genetics, vol. 9, no. 4, pp. 597-604, 2000.

[34] N. T. Leach and C. Jackson-Cook, “The application of spectral karyotyping (SKY) and fluorescent in situ hybridization (FISH) technology to determine the chromosomal content(s) of micronuclei," Mutation Research, vol. 495, no. 1-2, pp. 11-19, 2001.

[35] M. Fenech, "Cytokinesis-block micronucleus assay evolves into a "cytome" assay of chromosomal instability, mitotic dysfunction and cell death," Mutation Research, vol. 600, no. 1-2, pp. 58-66, 2006.

[36] M. Fenech, M. Kirsch-Volders, A. T. Natarajan et al., "Molecular mechanisms of micronucleus, nucleoplasmic bridge and nuclear bud formation in mammalian and human cells," Mutagenesis, vol. 26, no. 1, pp. 125-132, 2011.

[37] M. Kirsch-Volders, I. Decordier, A. Elhajouji, G. Plas, M. J. Aardema, and M. Fenech, "In vitro genotoxicity testing using the micronucleus assay in cell lines, human lymphocytes and 3D human skin models," Mutagenesis, vol. 26, no. 1, pp. 177-184, 2011. 
[38] V. Menzies, D. E. Lyon, R. K. Elswick Jr., N. L. McCain, and D. P. Gray, "Effects of guided imagery on biobehavioral factors in women with fibromyalgia," Journal of Behavioral Medicine, vol. 3, p. 3, 2012.

[39] F. Wolfe, H. A. Smythe, M. B. Yunus et al., "The American college of rheumatology 1990 criteria for the classification of fibromyalgia. Report of the multicenter criteria committee," Arthritis \& Rheumatism, vol. 33, no. 2, pp. 160-172, 1990.

[40] K. H. Jones, T. P. York, J. Juusola, A. Ferreira-Gonzalez, H. H. Maes, and C. Jackson-Cook, "Genetic and environmental influences on spontaneous micronuclei frequencies in children and adults: a twin study," Mutagenesis, vol. 26, no. 6, pp. 745752, 2011.

[41] M. Fenech and S. Bonassi, "The effect of age, gender, diet and lifestyle on DNA damage measured using micronucleus frequency in human peripheral blood lymphocytes," Mutagenesis, vol. 26, no. 1, pp. 43-49, 2011.

[42] J. Jiang, Linear and Generalized Linear Mixed Models and Their Applications, Springer, New York, NY, USA, 2007.

[43] M. Bibikova, B. Barnes, C. Tsan et al., "High density DNA methylation array with single CpG site resolution," Genomics, vol. 98, no. 4, pp. 288-295, 2011.

[44] P. Du, X. Zhang, C.-C. Huang et al., "Comparison of Betavalue and $\mathrm{M}$-value methods for quantifying methylation levels by microarray analysis," BMC Bioinformatics, vol. 11, article 587, 2010.

[45] J. Maksimovic, L. Gordon, and A. Oshlack, "SWAN: subsetquantile within array normalization for illumina infinium HumanMethylation450 BeadChips," Genome Biology, vol. 13, no. 6, article R44, 2012.

[46] S. Dedeurwaerder, M. Defrance, E. Calonne, H. Denis, C. Sotiriou, and F. Fuks, "Evaluation of the infinium methylation 450K technology," Epigenomics, vol. 3, no. 6, pp. 771-784, 2011.

[47] C. Zhu, Z. Xiong, Z. Zheng, Y. Chen, X. Qian, and X. Chen, "Association of serum gamma-glutamyltransferase with arterial stiffness in established coronary artery disease," Angiology, vol. 64, no. 1, pp. 15-20, 2013.

[48] M. Bucan, B. S. Abrahams, K. Wang et al., "Genome-wide analyses of exonic copy number variants in a family-based study point to novel autism susceptibility genes," PLoS Genetics, vol. 5, no. 6, Article ID e1000536, 2009.

[49] M. A. Pearen, J. G. Ryall, M. A. Maxwell, N. Ohkura, G. S. Lynch, and G. E. O. Muscat, "The orphan nuclear receptor, NOR-1, is a target of $\beta$-adrenergic signaling in skeletal muscle," Endocrinology, vol. 147, no. 11, pp. 5217-5227, 2006.

[50] Y. Niida and A. Yachie, "MAP7D2 is a brain expressing X-linked maternal imprinted gene in humans," Nature Proceedings, 2011, http://hdl.handle.net/10101/npre.2011.6684.1.

[51] R. C. Gentleman, V. J. Carey, D. M. Bates et al., "Bioconductor: open software development for computational biology and bioinformatics," Genome Biology, vol. 5, no. 10, article R80, 2004.

[52] G. K. Smyth, "Linear models and empirical bayes methods for assessing differential expression in microarray experiments," Statistical Applications in Genetics and Molecular Biology, vol. 3, no. 1, article 3, 2004.

[53] R Development Core Team, R: A Language and Environment for Statistical Computing, R Foundation for Statistical Computing, Vienna, Austria, 2011.

[54] Y. Benjamini and Y. Hochberg, "Controlling the false discovery rate: a practical and powerful approach to multiple testing," Journal of the Royal Statistical Society. Series B, vol. 57, no. 1, pp. 289-300, 1995.
[55] D. W. Huang, B. T. Sherman, Q. Tan et al., "The DAVID Gene Functional Classification Tool: a novel biological modulecentric algorithm to functionally analyze large gene lists," Genome Biology, vol. 8, no. 9, article R183, 2007.

[56] P. F. Kuan, S. Wang, X. Zhou, and H. Chu, "A statistical framework for Illumina DNA methylation arrays," Bioinformatics, vol. 26, no. 22, pp. 2849-2855, 2010.

[57] G. Iarmarcovai, M. Ceppi, A. Botta, T. Orsière, and S. Bonassi, "Micronuclei frequency in peripheral blood lymphocytes of cancer patients: a meta-analysis," Mutation Research, vol. 659, no. 3, pp. 274-283, 2008.

[58] E. Murgia, M. Ballardin, S. Bonassi, A. M. Rossi, and R. Barale, "Validation of micronuclei frequency in peripheral blood lymphocytes as early cancer risk biomarker in a nested case-control study," Mutation Research, vol. 639, no. 1-2, pp. 2734, 2008.

[59] L. Migliore, F. Coppedè, M. Fenech, and P. Thomas, "Association of micronucleus frequency with neurodegenerative diseases," Mutagenesis, vol. 26, no. 1, pp. 85-92, 2011.

[60] M. G. Andreassi, R. Barale, P. Iozzo, and E. Picano, “The association of micronucleus frequency with obesity, diabetes and cardiovascular disease," Mutagenesis, vol. 26, no. 1, pp. 77-83, 2011.

[61] I. Y. Iourov, Y. B. Yurov, and S. G. Vorsanova, "Mosaic X chromosome aneuploidy can help to explain the male-to-female ratio in autism," Medical Hypotheses, vol. 70, no. 2, p. 456, 2008.

[62] M. Fenech, A. El-Sohemy, L. Cahill et al., "Nutrigenetics and nutrigenomics: viewpoints on the current status and applications in nutrition research and practice," Journal of Nutrigenetics and Nutrigenomics, vol. 4, no. 2, pp. 69-89, 2011.

[63] R. L. Jirtle and M. K. Skinner, "Environmental epigenomics and disease susceptibility," Nature Reviews Genetics, vol. 8, no. 4, pp. 253-262, 2007.

[64] I. C. G. Weaver, N. Cervoni, F. A. Champagne et al., "Epigenetic programming by maternal behavior," Nature Neuroscience, vol. 7, no. 8, pp. 847-854, 2004.

[65] N. T. Leach, C. Rehder, K. Jensen, S. Holt, and C. JacksonCook, "Human chromosomes with shorter telomeres and large heterochromatin regions have a higher frequency of acquired somatic cell aneuploidy," Mechanisms of Ageing and Development, vol. 125, no. 8, pp. 563-573, 2004.

[66] N. T. Leach and C. Jackson-Cook, "Micronuclei with multiple copies of the $\mathrm{X}$ chromosome: do chromosomes replicate in micronuclei?" Mutation Research, vol. 554, no. 1-2, pp. 89-94, 2004.

[67] K. Brennan and J. M. Flanagan, "Is there a link between genomewide hypomethylation in blood and cancer risk?" Cancer Prevention Research, vol. 5, no. 12, pp. 1345-1357, 2012.

[68] P. van Damme, K. Hole, A. Pimenta-Marques et al., "NatF contributes to an evolutionary shift in protein N-terminal acetylation and is important for normal chromosome segregation," PLoS Genetics, vol. 7, no. 7, Article ID e1002169, 2011.

[69] M. Malcangio and V. Lessmann, "A common thread for pain and memory synapses? Brain-derived neurotrophic factor and trkB receptors," Trends in Pharmacological Sciences, vol. 24, no. 3, pp. 116-121, 2003.

[70] B. J. Kerr, E. J. Bradbury, D. L. H. Bennett et al., "Brain-derived neurotrophic factor modulates nociceptive sensory inputs and NMDA-evoked responses in the rat spinal cord," Journal of Neuroscience, vol. 19, no. 12, pp. 5138-5148, 1999. 
[71] S. Pezet, M. Malcangio, and S. B. McMahon, "BDNF: a neuromodulator in nociceptive pathways?" Brain Research Reviews, vol. 40, no. 1-3, pp. 240-249, 2002.

[72] K. Yamada and T. Nabeshima, "Brain-derived neurotrophic factor/TrkB signaling in memory processes," Journal Pharmacological Sciences, vol. 91, no. 4, pp. 267-270, 2003.

[73] M. F. Egan, M. Kojima, J. H. Callicott et al., "The BDNF val66met polymorphism affects activity-dependent secretion of BDNF and human memory and hippocampal function," Cell, vol. 112, no. 2, pp. 257-269, 2003.

[74] D. Kofink, M. P. Boks, H. T. Timmers, and M. J. Kas, "Epigenetic dynamics in psychiatric disorders: environmental programming of neurodevelopmental processes," Neuroscience \& Biobehavioral Reviews, vol. 37, no. 5, pp. 831-845, 2013.

[75] M. Weber, J. J. Davies, D. Wittig et al., "Chromosome-wide and promoter-specific analyses identify sites of differential DNA methylation in normal and transformed human cells," Nature Genetics, vol. 37, no. 8, pp. 853-862, 2005.

[76] K. Nakano, D. L. Boyle, and G. S. Firestein, "Regulation of DNA methylation in rheumatoid arthritis synoviocytes," The Journal of Immunology, vol. 190, no. 3, pp. 1297-1303, 2013.

[77] M. K. Shanmugam and G. Sethi, "Role of epigenetics in inflammation-associated diseases," Subcellular Biochemistry, vol. 61, pp. 627-657, 2012. 


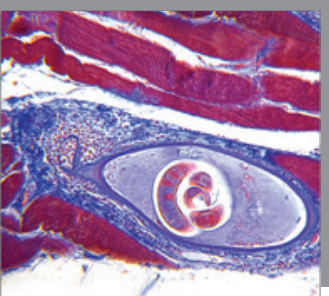

Gastroenterology

Research and Practice
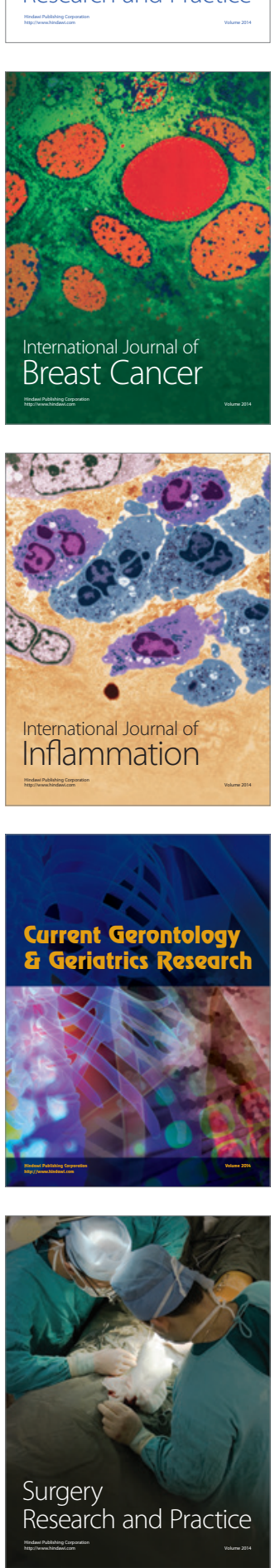

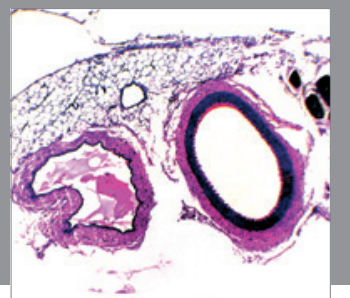

International Journal of Hypertension
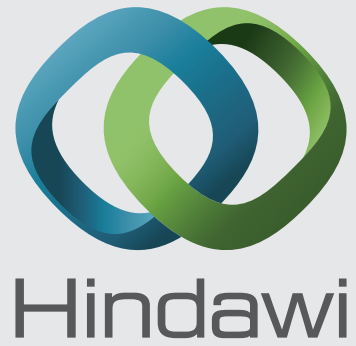

Submit your manuscripts at http://www.hindawi.com
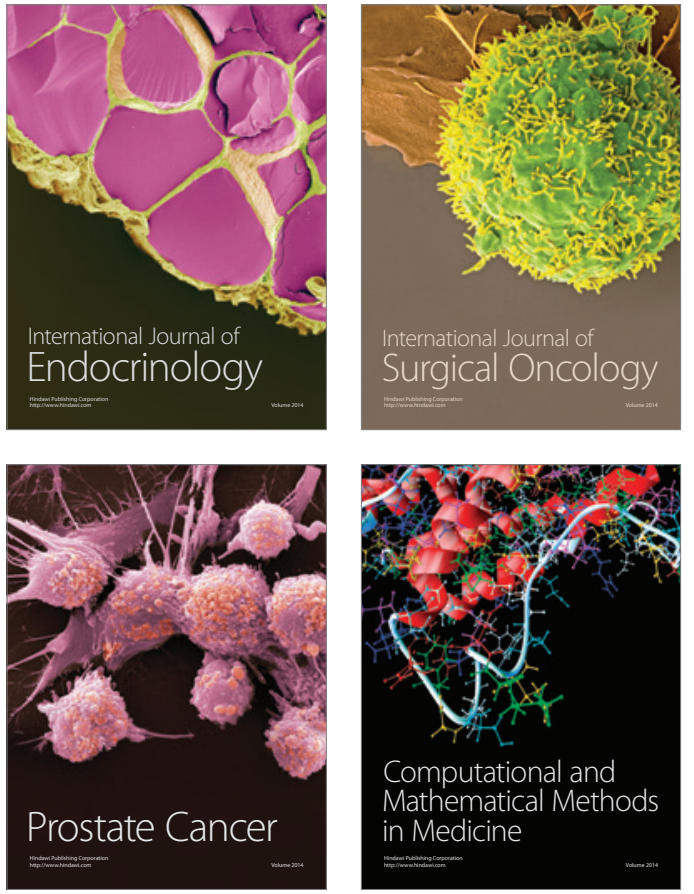
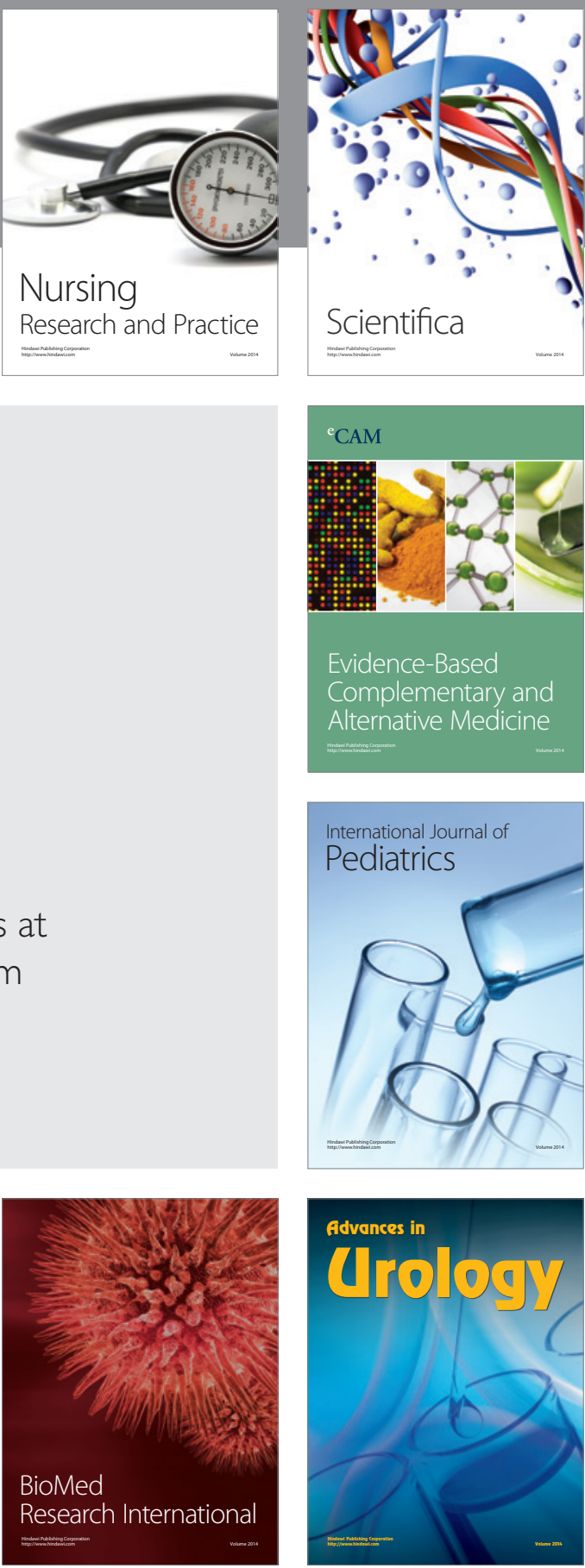

Nursing

Research and Practice

Scientifica

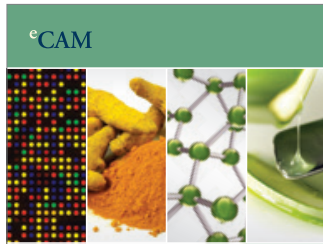

Evidence-Based

Complementary and Alternative Medicine
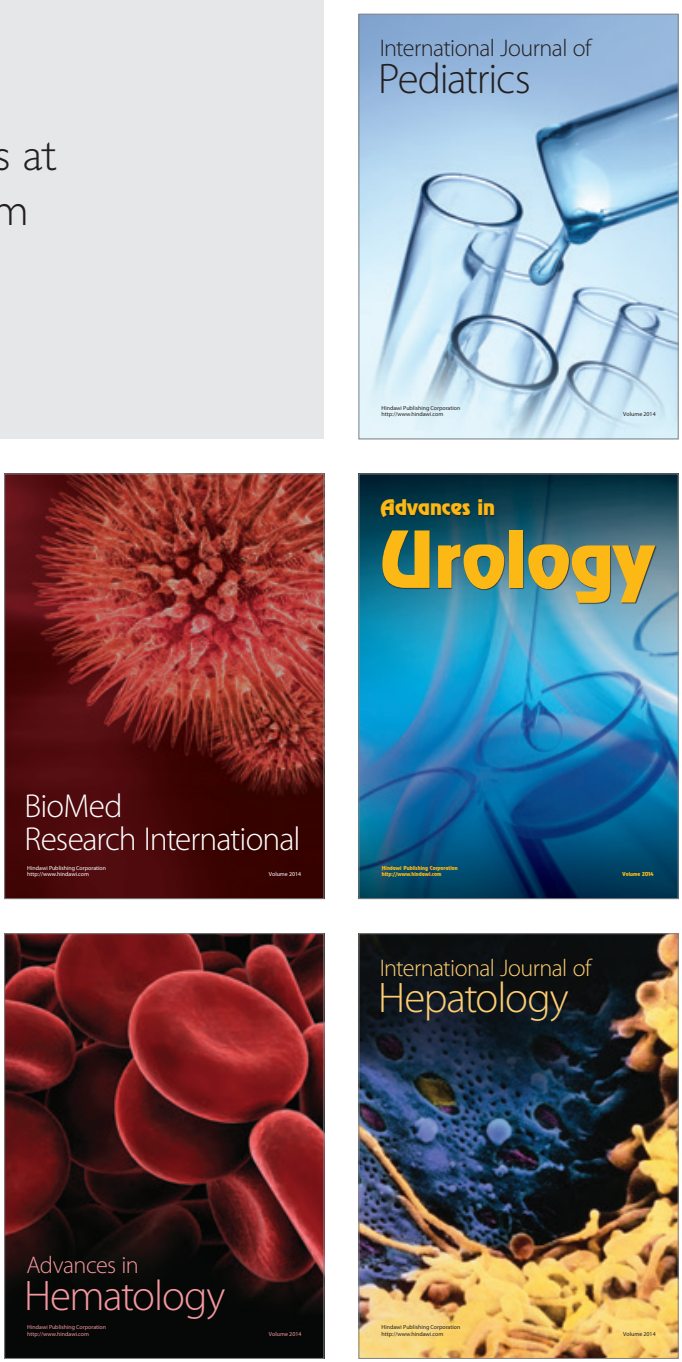